\title{
EL CREDO DEL “EVANGELIO DE LA VIDA"
}

\author{
THE CREED OF THE “GOSPEL OF LIFE”
}

P. Carlos Rosell De Almeida ${ }^{1}$

\section{RESUMEN}

El presente artículo: "El credo del Evangelio de la vida" tiene como intención mostrar de una manera sintética las enseñanzas esenciales de la Iglesia sobre el don sagrado de la vida humana. Para ello, se abordan los siguientes temas: el inicio de la vida humana; el matrimonio y la familia; la dignidad de toda vida humana independientemente de su estado; las condiciones básicas de desarrollo de la vida humana y la apertura del hombre a la eternidad. Para la exposición de estos puntos hacemos constantes referencias al Magisterio de la Iglesia, la primera defensora de los derechos básicos de la humanidad.

\section{Palabras clave}

Vida, matrimonio, familia, desarrollo, eternidad

\section{ABSTRACT}

This article: "The creed of the Gospel of life" is intended to show in a synthetic way the essential teachings of the Church on the sacred gift of human life. To do this, the following issues are addressed: the beginning of human life, marriage and family, the dignity of every human life regardless of their status, the basic conditions for the development of human life and man's openness to eternity. To explain these points we do constant references to the Magisterium of the Church, the first advocate of the basic rights of humanity.

\section{Keywords}

Life, marriage, family, development, eternity

Nos decía Juan Pablo II que "El Evangelio de la vida está en el centro del mensaje de Jesús. Acogido con amor cada día por la Iglesia, es anunciado con intrépida fidelidad como buena noticia a los hombres de todas las épocas y culturas". $\mathrm{Si}$ nosotros somos conscientes de que el "Evangelio de la vida" es parte esencial del mensaje del Señor, entonces, nos comprometeremos en ser portadores de este anuncio.

El "Evangelio de la vida" es el anuncio gozoso de que la vida humana es sagrada, es un don de Dios, es el primer derecho fundamental de la persona y condición para los otros derechos.
Al mismo tiempo, el "Evangelio de la vida" es un llamado para que custodiemos ese don. Por eso, he querido señalar en esta exposición cinco verdades sobre la vida humana que debemos de creer y anunciar como parte del mensaje evangelizador. A estas verdades las he llamado el "Credo del Evangelio de la vida".

\section{CREEMOS QUE LA VIDA HUMANA SE INICIA DESDE EL INSTANTE MISMO DE LA CONCEPCIÓN}

La primera verdad que debemos sostener con todas las fuerzas de nuestro ser es que la vida

\footnotetext{
Ingeniero Civil titulado (1993) y egresado de la Universidad Nacional de Ingeniería. Fue ordenado sacerdote para la Arquidiócesis de Lima el año 2004. Rector del Seminario Conciliar de Santo Toribio de Mogrovejo (Lima). Profesor en la Facultad de Teología Pontificia y Civil de Lima.

2 Juan Pablo II, Encíclica $N^{\circ} 1$.
} 
humana, toda vida humana, es sagrada desde el primer instante de su concepción. En el momento en que se realiza la concepción, nos encontramos ya con una verdadera persona humana. En efecto, existe una unidad psico-biológica $y$ espiritual, hay un auténtico código genético, existe una autonomía personal que ciertamente no es absoluta porque ningún hombre es independiente de los demás. Desde la teología hablamos de una unidad sustancial de cuerpo y alma, "corpore et anima unus", y donde el alma ha sido directamente infundida por Dios ${ }^{3}$.

Por más, que los partidarios de la "cultura de la muerte" se esfuercen por hacer malabares pseudo-técnicos, y cálculos artificiosos no se puede negar que desde el momento de la concepción nos encontramos con un "ser personal". Se trata de una vida intocable, un sujeto de derechos fundamentales dado que es persona. Asimismo, nuestra fe nos dice que esa persona es creada a imagen y semejanza de Dios (cf. Gn 1, 26-28), y por quien Cristo ha dado su vida (cf. Jn 10, 11.18).

Argumentar que recién se es persona luego de un tiempo de la concepción, o cuando el embrión empieza a "actuar", es un absurdo metafísico, dado que "el obrar sigue al ser", operari sequitur esse. Todo "ser vivo" está siempre en un proceso que se despliega en el tiempo, no se "es" a partir de un tiempo, sino desde el inicio de la concepción. En efecto, ya en el momento de la concepción se posee "el ser", se adquiere una "naturaleza". Además, afirmar que solo cuando pasa un tiempo después de la concepción se es persona, conduce a que sean algunos los que decidan cuando comienza a ser sujeto de derechos. Por tanto, la vida de este "ser" quedará en las manos de un determinado grupo que decidirá arbitrariamente si continúa o no su existencia.

Creer que la vida humana es sagrada desde su concepción, lleva a condenar el aborto directamente procurado y todo tipo de manipulación genética como son, por ejemplo, las técnicas de fecundación artificial. Son todos atentados directos contra la vida humana. El Beato Juan Pablo II afirmó con contundencia en su Encíclica Evangelium vitae lo siguiente: "Con la autoridad conferida por Cristo a Pedro y a sus Sucesores, en comunión con los Obispos de la Iglesia católica, confirmo que la eliminación directa y voluntaria de un ser humano inocente es siempre gravemente inmoral. Esta doctrina, fundamentada en aquella ley no escrita que cada hombre, a la luz de la razón, encuentra en el propio corazón (cf. $R m$ 2, 14-15), es corroborada por la Sagrada Escritura, transmitida por la Tradición de la Iglesia y enseñada por el Magisterio ordinario y universal"4.

Cuando la Iglesia dice "no" de manera absoluta a las prácticas intrínsecamente inmorales del aborto directamente procurado y todo tipo de manipulación genética, lo que hace es decir "sí" a la vida humana. Al defender la vida desde el primer instante de su concepción la Iglesia defiende la paz, dado que "todo delito contra la vida es un atentado contra la paz" 5 .

\section{CREEMOS QUE LA VIDA HUMANA EXIGE, COMO ÁMBITO DIGNO DE ACOGIDA, EL MATRIMONIO UNO E INDISOLUBLE}

Forma parte esencial del "Evangelio de la vida", el anuncio de la naturaleza $y$ valor sagrado del matrimonio. Defender el matrimonio es defender la vida humana. Todas las leyes que se promulguen contra esta institución, son siempre leyes intrínsecamente inmorales, contrarias a la vida y dignidad del mismo hombre. El matrimonio, en esencia, consiste en la unión entre un varón y una mujer. La misma etimología de matrimonio ${ }^{6}$ alude a la complementariedad de sexos. Es una institución que hunde sus raíces en la

\footnotetext{
"La Iglesia enseña que cada alma espiritual es directamente creada por Dios". CEC No 366

Juan Pablo II, Encíclica Evangelium vitae, № 57.

Pablo VI, Mensaje para la jornada mundial de la paz 1977.

La palabra matrimonio viene de matris: de la madre y munus: oficio. Por tanto, hace referencia a la maternidad. "En latín se usan varios términos para designar el matrimonio: Matrimonium (matris, munium) que se deriva de mater (madre). Se dan varias explicaciones del origen de esta palabra. Indicaría el efecto o fin principal del matrimonio, a saber la procreación que hace fecunda a la madre...". P. Adnés, El matrimonio, Barcelona 1969, p.138.
} 
creación del hombre. Aunque para nosotros, los católicos, a partir de Cristo sea sacramento (cf. Ef 5, 32), es de ley natural que no es posible considerar verdadero matrimonio a la unión entre personas del mismo sexo.

La vida humana necesita ser recibida en el matrimonio, como fruto de un verdadero "acto conyugal". ¿Qué es el acto conyugal? Es el acto por el cual los esposos se unen sexualmente de manera natural en pleno respeto a las leyes inscritas por Dios en ellos. Este acto debe expresar el amor conyugal que tiene las siguientes características: "Es, ante todo, un amor humano... Es un amor total... Es un amor fiel y exclusivo hasta la muerte. Es un amor fecundo, que no se agota en la comunión entre los esposos sino que está destinado a prolongarse suscitando nuevas vidas"'. Tenemos aquí un gran reto para proclamar el "Evangelio de la vida": que los esposos vivan el amor conyugal. La inseparabilidad entre lo unitivo y lo procreativo expresan la belleza del amor conyugal: "unidad de dos" abiertos a la vida.

El "Evangelio de la vida" lleva consigo la preparación de los jóvenes para que acepten el matrimonio según su propia naturaleza. La preparación al matrimonio no es un "acto burocrático", un mero trámite, sino un hecho de enorme responsabilidad en la que se está jugando el futuro de esa persona. El gran porcentaje de matrimonios separados, o que no viven el amor conyugal, al hacer uso de los métodos anticonceptivos, es un llamado de atención serio para revertir esa situación. Uno de los aspectos fundamentales es hablar a los jóvenes sobre la belleza del amor conyugal.

El matrimonio es el ámbito digno donde se engendra una vida, pero no solo se trata de la transmisión de la vida sino también de educar esa vida. Los padres de familia son portadores del "Evangelio de la vida", cuando tras engendrar a sus hijos, los educan. "Puesto que los padres han dado la vida a los hijos, están gravemente obligados a la educación de la prole $y$, por tanto, ellos son los primeros y principales educadores. Este deber de la educación familiar es de tanta trascendencia que, cuando falta, difícilmente puede suplirse" ${ }^{\text {. }}$. Pero, ¿en qué consiste educar a los hijos? Consiste en formarlos integralmente, en moldear sus vidas en la verdad y el amor. El "Evangelio de la vida" exige a los padres de familia que "formen" a sus hijos, no solo preocupándose para que reciban conocimientos y una vasta cultura, sino cuidando su formación integral, forjando en ellos las virtudes. Educar a los hijos en sólidos principios morales es la mejor herencia que pueden dejar los padres y una contribución inestimable para el "Evangelio de la vida".

Cuando hablamos del matrimonio necesariamente debemos de referirnos a la familia. El "Evangelio de la vida" proclama el valor sagrado de la familia, santuario de la vida humana, ámbito fundamental donde se desarrollan las relaciones de paternidad, maternidad, filiación y fraternidad. Decía Benedicto XVI: "La familia es un fundamento indispensable en la sociedad y los pueblos, así como un bien insustituible para los hijos, dignos de venir a la vida como fruto del amor, de la donación total y generosa de los padres. Como puso de manifiesto Jesús honrando a la Virgen María y a San José, la familia ocupa un lugar primario en la educación de la persona. Es una verdadera escuela de humanidad y valores perennes. Nadie se ha dado el ser a sí mismo. Hemos recibido de otros la vida, que se desarrolla y madura con las verdades y valores que aprendemos en la relación y comunión con los demás. En este sentido, la familia fundada en el matrimonio indisoluble entre un hombre y una mujer expresa esta dimensión relacional, filial y comunitaria, y es el ámbito donde el hombre puede nacer con dignidad, crecer y desarrollarse de un modo integral"'.

\section{CREEMOS QUE LA VIDA HUMANA, SEA CUAL SEA SU SITUACIÓN, SIEMPRE ES VALIOSA}

Hoy en día es común presentar la vida humana de manera discriminada. ¿A qué me refiero? Al

\footnotetext{
Pablo VI, Encíclica Humanae vitae, № 9.

Concilio Vaticano II, Declaración Gravissimum educationis, $\mathrm{N}^{\circ} 3$.

Benedicto XVI, Mensaje de Su Santidad Benedicto XVI en el VI Encuentro Mundial de las Familias, 2012.
} 
hecho de que no se considera que toda vida humana sea igual. Dicho en otras palabras, existe en al mundo de hoy la idea errada que hay vidas humanas de menor calidad. Frente a esta postura, la Iglesia alza su voz y defiende toda vida humana, pues para Dios no hay vidas de "segunda categoría". Por eso, condena la eutanasia. Juan Pablo señaló a este respecto: "De acuerdo con el Magisterio de mis Predecesores y en comunión con los Obispos de la Iglesia católica, confirmo que la eutanasia es una grave violación de la Ley de Dios, en cuanto eliminación deliberada y moralmente inaceptable de una persona humana" ${ }^{10}$.

Campea en la sociedad una corriente de pensamiento que ve la vida humana como valiosa solo en relación con su eficacia, o con su capacidad de adquirir bienes materiales. La "cultura de la muerte" ha puesto también sus miras asesinas sobre los enfermos, los ancianos, y los que sufren limitaciones físicas o mentales, es decir, los discapacitados. Esto se manifiesta en sociedades "caracterizadas por una mentalidad eficientista que presenta el creciente número de personas ancianas $y$ debilitadas como algo demasiado gravoso $e$ insoportable"11.

Las personas enfermas, ancianas, o discapacitadas, no son menos personas que nadie. Cada persona es única e irrepetible, posee un valor intrínseco en sí misma. El lugar de las personas enfermas, ancianas, o limitadas en la sociedad es fundamental, porque gracias a ellas nosotros vemos nuestra verdad: todos somos seres limitados y necesitamos de los demás. Valorar cada vida humana, nos lleva a acoger a todos y forjar una sociedad donde nadie se sienta maltratado en su dignidad de persona. Más aún, la Iglesia nos enseña que los enfermos, los ancianos, las personas limitadas son los preferidos del Señor. Podemos decir que "los pobres y los abandonados, los enfermos, los marginados son la carne de Cristo" 12 .

En este sentido, el "Evangelio de la vida" exige que los centros de salud, los hospitales, los asilos, etc., sean dignos de las personas. Urge un llamado a todos los responsables sanitarios para que acojan a los enfermos y ancianos como verdaderas personas. Debemos unirnos para que la atención a ellos sea siempre digna. Si nos esforzamos para que las personas que sufren enfermedades, limitaciones o el peso de la edad, encuentren un espacio de amor y comprensión, estaremos contribuyendo enormemente a combatir todo intento de promover la eutanasia.

En relación con esta verdad de que no hay vida humana de "segunda categoría", debo de dedicar también unas palabras, al problema del "racismo". Es totalmente contrario al "Evangelio de la vida", discriminar a una persona por su raza. La vida humana no se califica por la raza, sino en símisma. "Elprejuicio racista, que niega la igual dignidad de todos los miembros de la familia humana y blasfema de su Creador, solo puede ser combatido donde nace, es decir, en el corazón del hombre. Del corazón brotan los comportamientos justos o injustos, según que el hombre se abra a la voluntad de Dios, en el orden natural y en su Palabra viva, o se encierre en sí mismo y en su egoísmo, dictado por el miedo o por el instinto de dominio. Es la visión del otro que es preciso purificar. Alimentar concepciones y fomentar actitudes racistas es un pecado contra la enseñanza específica de Cristo, para quien el "prójimo» no es solamente el hombre de mi tribu, de mi ambiente, de mi religión o de mi nación, es todo ser humano que encuentro en mi camino"13.

\section{CREEMOS QUE LA VIDA HUMANA EXIGE DE PARTE DE LA SOCIEDAD, UNAS CONDICIONES BÁSICAS DE DESARROLLO}

No basta combatir el aborto y la eutanasia para afirmar que anunciamos el "Evangelio de la vida". Debemos ser conscientes que la vida humana se desarrolla en la sociedad, y ésta tiene que brindar una serie de condiciones para una vida realmente digna. En la Constitución

\footnotetext{
10 Juan Pablo II, Encíclica Evangelium vitae, № 65.

11 Juan Pablo II, Encíclica Evangelium vitae, $N^{\circ} 64$.

12 Francisco, Homilía en la canonización de la Madre Lupita, Madre Laura y 800 mártires italianos, 12/05/2013.

13 Pontificio Consejo Iustitia Et Pax, La Iglesia ante el racismo, para una sociedad más fraterna, № 24.
} 
Gaudium spes, redactada en el Concilio Vaticano II, se indicó la necesidad de que en la sociedad se propugne la consecución del bien común que es "el conjunto de condiciones de la vida social que hacen posible a las asociaciones y a cada uno de sus miembros el logro más pleno y más fácil de la propia perfección"14. Gracias, al bien común, cada persona humana puede tener una vida digna.

Estimo que existen tres puntos fundamentales que afectan directamente la vida humana en la sociedad y que es necesario asumir como verdaderos retos. En primer lugar, está la recta visión de la sexualidad. El hombre es un ser sexuado, existe o como varón o como mujer. La sexualidad es una dimensión de la persona humana. Se atenta contra la vida, cuando se deforma el ejercicio de la sexualidad. Hoy en día, la pornografía es un medio promovido por "la cultura de la muerte" para destruir la vida humana. ¿Por qué? Dado que al cosificar el sexo, lo convierte en mercancía barata, un mero instrumento de goce, sin referencia alguna a la donación, en definitiva al amor. Las consecuencias saltan a la vida, la difusión de un sexo sin límites, engendra hombres y mujeres egoístas que no respetan la vida de los demás. Se han vuelto esclavos de sus instintos sexuales, sin capacidad para amar. "La Iglesia, que se interesa por el verdadero desarrollo del hombre, exhorta a este a que respete los valores humanos también en el ejercicio de la sexualidad: ésta no puede quedar reducida a un mero hecho hedonista y lúdico, del mismo modo que la educación sexual no se puede limitar a una instrucción técnica, con la única preocupación de proteger a los interesados de eventuales contagios o del "riego de procrear" 15. La única manera de humanizar la sexualidad, es vivir la castidad. Por eso, "el Evangelio de la vida", anuncia el valor de esta virtud, la cual "significa la integración de la sexualidad en la persona. Entraña el aprendizaje del dominio personal." 16 .

Quien anuncia el "Evangelio de la vida" se compromete a purificar el ambiente salvándolo del predominio hedonista. Como sabemos el hedonismo promueve la consecución del placer, en especial de tipo sexual, como primer valor. Enfrentarse a esta corriente lleva consigo medidas concretas como evitar el uso de los medios de comunicación que promueven un estilo de vida inmoral. Hay que humanizar las vías de información para que se transmitan contenidos que "formen" y no "deformen". La libertad de expresión no es una carta abierta para transmitir todo tipo de contenidos sin límite moral alguno. Más bien, "el recto ejercicio de este derecho exige que, en cuanto a su contenido, la comunicación sea siempre verdadera e íntegra, salvadas la justicia y la caridad; además, en cuanto al modo, ha de ser honesta y conveniente, es decir, debe respetar escrupulosamente las leyes morales, los derechos legítimos y la dignidad del hombre, tanto en la búsqueda de la noticia como en su divulgación, ya que no todo conocimiento aprovecha, pero la caridad es constructiva (1 Cor 8, 1)"17.

Un segundo punto que la sociedad tiene que proteger y promover es el trabajo digno. En cierta manera, se "mata" a una persona cuando no se le da la oportunidad de trabajar o su trabajo no corresponde con la dignidad personal. Anunciar el "Evangelio de la vida" implica colaborar para que nadie en nuestra sociedad carezca de un trabajo digno. ¿Qué es un trabajo digno? Escuchemos la magnífica explicación de Benedicto XVI: "Significa un trabajo que, en cualquier sociedad, sea expresión de la dignidad esencial de todo hombre o mujer: un trabajo libremente elegido, que asocie efectivamente a los trabajadores, hombres y mujeres, al desarrollo de su comunidad; un trabajo que, de este modo, haga que los trabajadores sean respetados, evitando toda discriminación; un trabajo que permita satisfacer las necesidades de las familias y escolarizar a los hijos sin que se vean obligados a trabajar; un trabajo que consienta a los trabajadores organizarse libremente y hacer oír su voz; un trabajo que deje espacio para reencontrarse adecuadamente con las

14 Concilio Vaticano II, Constitución pastoral Gaudium et spes $\mathrm{N}^{\circ} 26$.

15 Benedicto XVI, Encíclica Caritas in veritate, № 44.

16 CEC N 2395.

17 Concilio Vaticano II, Declaración Inter mirifica, N 5 
propias raíces en el ámbito personal, familiar $y$ espiritual; un trabajo que asegure una condición digna a los trabajadores que llegan a la jubilación"18.

No exagero si digo que hoy se atenta contra el "Evangelio de la vida" con leyes labores que son verdaderamente "esclavizantes". Hoy prolifera la tercerización, pero, algunas de las empresas que ofrecen estos servicios no dan condiciones dignas para que sus trabajadores. Los sueldos que ofrecen son en muchos casos injustos. La vida, volvamos a decirlo, también se defiende forjando condiciones laborales dignas para los trabajadores.

El tercer punto que considero importante en relación con las condiciones para una vida humana digna es la libertad religiosa, es decir, el derecho de profesar la religión que en consciencia uno cree verdadera. "Esta libertad consiste en que todos los hombres han de estar inmunes de coacción, tanto por parte de individuos como de grupos sociales $y$ de cualquier potestad humana, y esto de tal manera que, en materia religiosa, ni se obligue a nadie a obrar contra su conciencia, ni se le impida que actúe conforme a ella en privado y en público, solo o asociado con otros, dentro de los límites debidos"19.

Nosotros, los católicos, debemos de defender el derecho que tenemos de profesar nuestra fe $y$, al mismo tiempo, tender lazos de amistad con las otras religiones. Podemos y debemos establecer relaciones con otros credos en favor del "Evangelio de la Vida". En el tema de la defensa de la vida humana, en líneas generales, estamos de acuerdo con las grandes religiones.

papa Francisco, en la Santa Misa de inauguración de su pontificado, enseñó que todos, independientemente de nuestro credo religioso, tenemos la vocación de custodiar la vida humana: "La vocación de custodiar no solo nos atañe a nosotros, los cristianos, sino que tiene una dimensión que antecede y que es simplemente humana, corresponde a todos. Es custodiar toda la creación, la belleza de la creación, como se nos dice en el libro del Génesis y como nos muestra san Francisco de Asís: es tener respeto por todas las criaturas de Dios y por el entorno en el que vivimos. Es custodiar a la gente, el preocuparse por todos, por cada uno, con amor, especialmente por los niños, los ancianos, quienes son más frágiles $y$ que a menudo se quedan en la periferia de nuestro corazón. Es preocuparse uno del otro en la familia: los cónyuges se guardan recíprocamente y luego, como padres, cuidan de los hijos, y con el tiempo, también los hijos se convertirán en cuidadores de sus padres"20.

\section{CREEMOS QUE LA VIDA HUMANA NO SE LIMITA A ESTE MUNDO, SINO QUE ESTÁ ABIERTA A LA ETERNIDAD}

No podemos referirnos a la vida humana sin apelar a nuestra fe. Nosotros, los católicos, como San Pablo afirmamos: "Para mí, la vida es Cristo, y la muerte, una ganancia" $(\boldsymbol{F l p ~ 1 , ~ 2 1 ) . ~ P o r ~ e s o , ~ e l ~ " E v a n g e l i o ~ d e ~ l a ~ v i d a " ~}$ exige mostrar a los demás que la vida humana adquiere un sentido pleno solo desde la comunión con Cristo. Todo tiene sentido desde la fe en Cristo muerto por nuestros pecados pero resucitado para traernos la verdadera vida (cf. $R m$ 4, 25). Cristo lo ilumina todo y nos anima con el Espíritu Santo para ser verdadero portadores del "Evangelio de la vida".

Nuestra fe en Cristo nos enseña que cada hombre está llamado por Dios uno y trino a la comunión eterna con él. La sed de eternidad, que está en el corazón de todo hombre, encuentra su respuesta en la vida eterna que Dios Padre nos ofrece en su Hijo, por el don del Espíritu Santo. "En Jesús, "Palabra de vida", se anuncia y comunica la vida divina y eterna. Gracias a este anuncio y a este don, la vida física y espiritual del hombre, incluida su etapa terrena, encuentra plenitud de valor y significado: en efecto, la vida divina y eterna es el fin al que está orientado y llamado el

\footnotetext{
18 Benedicto XVI, Encíclica Caritas in veritate No 63.

19 Concilio Vaticano II, Declaración Dignitatis humanae, No 3

20 Francisco, Misa de inauguración del pontificado, 19-03-2013.
} 
hombre que vive en este mundo. El Evangelio de la vida abarca así todo lo que la misma experiencia y la razón humana dicen sobre el valor de la vida, lo acoge, lo eleva y lo lleva a término"21.

Con una mirada meramente horizontal no encontramos sentido a muchas cosas desconcertantes que ocurren en el mundo. Pero, la mirada de la fe es trascendente, nos habla de la "vida eterna", del cielo, de una eternidad feliz en "Cristo Jesús" (cf. 1 Co 2, 9; Flp 3, 20). Esto me lleva a señalar la importancia de vivir en "gracia de Dios". Es decir, a vivir con el Señor. En cambio, la vida de pecado conduce al hombre a una falta de paz interior, un vacío existencial, una felicidad aparente. La fe nos enseña que quien muere en pecado mortal, sin arrepentimiento, pierde el cielo (cf. Mt 25, 31 ss.; 1 Co 6, 9 ss.; Ga 5, 21; Ef 5,5). El infierno es la "no vida" (cf. Ap 21, 27). Por eso, también anunciamos el "Evangelio de la vida" cuando hacemos que una persona salga del pecado, se encuentre con Cristo, único Redentor del mundo, y de esa manera alcancen la salvación.

\section{CONCLUSIONES}

El "Evangelio de la vida" es un auténtico reto. Se trata de un reto de anuncio y denuncia. De anuncio, porque cada uno de nosotros está llamado a proclamar la belleza de la vida humana Esto lleva consigo, mostrar la verdad de la concepción humana, del matrimonio, de la familia, del carácter sagrado de la sexualidad. Además, el "Evangelio de la vida" es un reto de denuncia. Si anunciamos el don inestimable de la vida humana, entonces, necesariamente y sin ningún tipo de temores, debemos oponernos a todo lo que ataca la vida de cada persona humana. Esto es: el aborto, la manipulación de embriones, la unión entre personas del mismo sexo, la eutanasia, la discriminación racial, la pornografía, etc. El motivo de esta denuncia no es en ningún caso ideológico, sino que es una defensa sobre la verdad del hombre. Conviene, enfatizar esta idea: cuando se anuncia el "Evangelio de la vida" se está defendiendo a cada hombre. En especial, la vida humana del más desprotegido de todos: el concebido no nacido. Pero también, la vida del enfermo, del anciano, del cónyuge, del trabajador, en definitiva, de todo hombre.

Quisiera concluir con una palabras del Papa Francisco y que vienen a ser una invitación a contemplar a ese Dios de la vida y del amor, que nos pide un profundo respeto hacia toda vida humana. A este respecto, el Papa nos indica: "Queridos hermanos y hermanas, miremos a Dios como al Dios de la vida, miremos su ley, el mensaje del Evangelio, como una senda de libertad y de vida. El Dios vivo nos hace libres. Digamos sí al amor y no al egoísmo, digamos sí a la vida y no a la muerte, digamos sí a la libertad y no a la esclavitud de tantos ídolos de nuestro tiempo; en una palabra, digamos sí a Dios, que es amor, vida y libertad, y nunca defrauda (cf. 1 Jn 4,8, Jn 11,25, Jn 8,32), a Dios que es el Viviente y el Misericordioso. Sólo la fe en el Dios vivo nos salva; en el Dios que en Jesucristo nos ha dado su vida con el don del Espíritu Santo y nos hace vivir como verdaderos hijos de Dios por su misericordia. Esta fe nos hace libres y felices. Pidamos a María, Madre de la Vida, que nos ayude a acoger y dar testimonio siempre del "Evangelio de la Vida». Así sea".

21 Juan Pablo II, Encíclica Evangelium vitae, № 30.

22 Francisco, Homilía en la Santa Misa de la jornada Evangelium vitae, 16-06-2013. 


\section{REFERENCIAS}

Adnés, P. (1969) El matrimonio. Barcelona: Herder.

Auer, J. (1977) Los sacramentos en la Iglesia. Barcelona: Herder.

Benedicto XVI (2012) Mensaje de Su Santidad Benedicto XVI en el VI Encuentro Mundial de las Familias.

Benedicto XVI (2009) Encíclica Caritas in veritate.

Catecismo de la Iglesia Católica (1992).

Concilio Vaticano II (1963) Declaración Inter mirifica.

Concilio Vaticano II (1965) Declaración Gravissimum educationis.

Concilio Vaticano II (1965) Declaración Dignitatis humanae.

Concilio Vaticano II (1965) Constitución pastoral Gaudium et spes.

Juan Pablo II (1995) Encíclica Evangelium vitae.

Pablo VI (1977) Mensaje para la jornada mundial de la paz 1977.

Pontificio Consejo Iustitia Et Pax (1988) La Iglesia ante el racismo, para una sociedad más fraterna. Sarmiento, A. (2001) El matrimonio cristiano. Pamplona: EUNSA. 